\title{
The partially shared values and small functions for meromorphic functions in a $k$-punctured complex plane
}

\author{
Hong Yan $\mathrm{Xu}^{1^{*}} \mathbb{D}$, Yong Ming $\mathrm{Li}^{1}$ and Shan $\mathrm{Liu}^{2}$
}

${ }^{\text {"Correspondence: }}$
xhyhhh@126.com
'School of Mathematics and
Computer Science, Shangrao
Normal University, Shangrao, China
Full list of author information is
available at the end of the article

available at the end of the article

\begin{abstract}
The main aim of this article is to discuss the uniqueness of meromorphic functions partially sharing some values and small functions in a k-punctured complex plane $\Omega$. We proved the following: Let $f_{1}, f_{2}$ be two admissible meromorphic functions in $\Omega$ and $\alpha_{j}(j=1,2, \ldots, l)$ be $/(\geq 5)$ distinct small functions with respect to $f$ and $g$. If $\widetilde{E}\left(\alpha_{j}, \Omega, f_{1}\right) \subseteq \widetilde{E}\left(\alpha_{j}, \Omega, f_{2}\right)(j=1,2, \ldots, l)$ and

$$
\liminf _{r \rightarrow+\infty} \frac{\sum_{j=1}^{l} \bar{N}_{0}\left(r, \frac{1}{f_{1}-\alpha_{j}}\right)}{\sum_{j=1}^{l} \bar{N}_{0}\left(r, \frac{1}{f_{2}-\alpha_{j}}\right)}>\frac{5}{2 l-5^{\prime}}
$$
\end{abstract}

then $f_{1} \equiv f_{2}$. Our results are some improvements and extension of previous theorems given by Cao-Yi and Ge-Wu.

MSC: Primary 30D30; secondary 30D35

Keywords: Meromorphic function; Partially sharing; Small function; k-punctured

\section{Introduction}

This article is devoted to the study of uniqueness of functions which are meromorphic in a multiply-connected domain- $k$-punctured complex plane $\Omega$. In 1920 s, Nevanlinna gave the definition of characterized function $T(r, f)$ of meromorphic function and established the famous first and second main theorem, lemma on the logarithmic derivatives etc. of Nevalinna theory (see Hayman [1], Yang [2] and Yi and Yang [3]). Nowadays, Nevanlinna theory is a powerful tool in studying the properties of meromorphic functions in the fields of complex analysis. By applying this theory, the following well-known five-value theorem was given by Nevanlinna [4].

Theorem A (see [4]) Iff and $g$ are two nonconstant meromorphic functions that share five distinct values $a_{1}, a_{2}, a_{3}, a_{4}, a_{5} I M$ in $X=\mathbb{C}$, then $f(z) \equiv g(z)$.

Nevanlinna [4] also pointed out the following question.

Question A (see [4]) Does Theorem A still hold if the five distinct values $a_{1}, a_{2}, a_{3}, a_{4}, a_{5}$ are replaced by five distinct small functions $\alpha_{j}(j=1,2, \ldots, 5)$ ?

(c) The Author(s) 2019. This article is distributed under the terms of the Creative Commons Attribution 4.0 International License (http://creativecommons.org/licenses/by/4.0/), which permits unrestricted use, distribution, and reproduction in any medium, provided you give appropriate credit to the original author(s) and the source, provide a link to the Creative Commons license, and indicate if changes were made. 
Around Theorem A and Question A, the value distribution theory of meromorphic functions occupies one of the central places in complex analysis. Moreover, it is always an interesting topic how to extend and improve some important uniqueness theorems in the complex plane to the subset $\mathbb{X}$ (including the unit disc, the angular domain, the annulus, etc.). Many scholars have paid significant attention to this topic and obtained lots of meaningful and important results (see [3, 5-9]). For example, Fang [10] in 1999 proved the five-value theorem for meromorphic functions in the unit disc; Zheng [11] in 2003 obtained the five-value theorem for meromorphic functions in an angular domain; Cao, $\mathrm{Yi}$, and $\mathrm{Xu}$ [12] in 2009 gave the five-value theorem for meromorphic functions in the annuli with the help of the Nevanlinna theory for meromorphic functions on annuli given by Khrystiyanyn and Kondratyuk [13, 14], or [15] in 2005, or [16] in 2004 (see [12]), etc. including [3, 10, 11, 17-22]; Yi and Yang, Lahiri, and Xu improved a series of uniqueness theorems about weight-shared and partially shared (see [3,23-26]); there are a series of beautiful and important results related to Question A (see [27-32]). Especially, Yi [31] gave a positive answer to Question A and extended the five-value theorem to the case of sharing five distinct small functions.

Theorem B ([31] The five small functions theorem) Letf and $g$ be two nonconstant meromorphic functions in a complex plane $\mathbb{C}$ and $a_{j}(j=1,2,3,4,5)$ be five distinct small functions with respect to $f$ and $g$. If $f$ and $g$ share $a_{j}(j=1,2,3,4,5)$ IM in $\mathbb{C}$, then $f \equiv g$.

In 2016, the authors investigated the uniqueness of meromorphic functions sharing some finite sets in a special multiply-connected region- $k$-punctured complex planeand obtained an analog of Nevanlinna's famous five-value theorem for meromorphic functions $f$ and $g$ in a $k$-punctured complex plane $[33,34]$. To state the result, some basic notations and a definition about $k$-punctured complex plane should be introduced as follows, which can be found in [35].

For $k$ distinct points $c_{j} \in \mathbb{C}, j \in\{1,2, \ldots, k\}, \Omega=\mathbb{C} \backslash \bigcup_{j=1}^{k}\left\{c_{j}\right\}$ can be called a $k$-punctured complex plane. Of course, the annulus is a special $k$-punctured plane as $k=1$. Let $k \geq 2$, $d=\frac{1}{2} \min \left\{\left|c_{s}-c_{j}\right|: j \neq s\right\}$, and $r_{0}=\frac{1}{d}+\max \left\{\left|c_{j}\right|: j \in\{1,2, \ldots, k\}\right\}$, thus it yields that $\frac{1}{r_{0}}<d$,

$$
\bar{D}_{1 / r_{0}}\left(c_{j}\right) \cap \bar{D}_{1 / r_{0}}\left(c_{s}\right)=\emptyset \text { for } j \neq s
$$

and

$$
\bar{D}_{1 / r_{0}}\left(c_{j}\right) \subset D_{r_{0}}(0) \text { for } j \in\{1,2, \ldots, k\}
$$

where $D_{\delta}(c)=\{z:|z-c|<\delta\}$ and $\bar{D}_{\delta}(c)=\{z:|z-c| \leq \delta\}$. Define

$$
\Omega_{r}=D_{r}(0) \backslash \bigcup_{j=1}^{k} \bar{D}_{1 / r}\left(c_{j}\right) \quad \text { for any } r \geq r_{0} .
$$

Thus, it follows that $\Omega_{r} \supset \Omega_{r_{0}}$ for $r_{0}<r \leq+\infty$. Obviously, $\Omega_{r}$ is a multiple connected and $k+1$ connected region. 
For a meromorphic function $f$ in the $k$-punctured plane $\Omega$ and $r_{0} \leq r<+\infty$, let $n_{0}(r, f)$ denote the counting function of its poles in $\bar{\Omega}_{r}$, and

$$
\begin{aligned}
N_{0}(r, f)= & \int_{r_{0}}^{r} \frac{n_{0}(t, f)}{t} d t, \\
m_{0}(r, f)= & \frac{1}{2 \pi} \int_{0}^{2 \pi} \log ^{+}\left|f\left(r e^{i \theta}\right)\right| d \theta+\frac{1}{2 \pi} \sum_{j=1}^{m} \int_{0}^{2 \pi} \log ^{+}\left|f\left(c_{j}+\frac{1}{r} e^{i \theta}\right)\right| d \theta \\
& -\frac{1}{2 \pi} \int_{0}^{2 \pi} \log ^{+}\left|f\left(r_{0} e^{i \theta}\right)\right| d \theta-\frac{1}{2 \pi} \sum_{j=1}^{m} \int_{0}^{2 \pi} \log ^{+}\left|f\left(c_{j}+\frac{1}{r_{0}} e^{i \theta}\right)\right| d \theta,
\end{aligned}
$$

where $\log ^{+} x=\max \{\log x, 0\}$, then

$$
T_{0}(r, f)=m_{0}(r, f)+N_{0}(r, f)
$$

is called the Nevanlinna characteristic of $f$ in the $k$-punctured complex plane. Besides, we use $S(r, f)$ to denote any quantity satisfying $S(r, f)=o\left(T_{0}(r, f)\right)$ for all $r$ outside a possible exceptional set $E$ of finite linear measure.

Definition 1.1 (see [33]) Let $f$ be a nonconstant meromorphic function in a $k$-punctured plane $\Omega$. The function $f$ is called admissible in a $k$-punctured plane $\Omega$ provided that

$$
\limsup _{r \rightarrow+\infty} \frac{T_{0}(r, f)}{\log r}=+\infty, \quad r_{0} \leq r<+\infty .
$$

Remark 1.1 (see [33]) From Theorem 5 in [35], a meromorphic function $f$ in a $k$ punctured plane is rational if $f$ satisfies

$$
\limsup _{r \rightarrow+\infty} \frac{T_{0}(r, f)}{\log r}<+\infty, \quad r_{0} \leq r<+\infty .
$$

Theorem C (see [33, Theorem 3.1]) Let $f$ and $g$ be two admissible meromorphic functions in $\Omega$; iff , $g$ share five distinct values $a_{1}, a_{2}, a_{3}, a_{4}, a_{5} \operatorname{IM}$ in $\Omega$, then $f(z) \equiv g(z)$.

\section{Results}

The purpose of this article is to extend and improve some uniqueness results (including Theorems A-C) to a special multiply-connected region-k-punctured complex plane.

By relaxing the form of sharing values $I M$ to the partially sharing in Theorem $\mathrm{C}$, we obtain the first result of this article, which is an improvement of Theorem C.

Theorem 2.1 Let $f_{1}, f_{2}$ be two admissible meromorphic functions in $\Omega, a_{1}, a_{2}, \ldots, a_{l}$ be $l(\geq$ 5) distinct values. If $\widetilde{E}\left(a_{j}, \Omega, f_{1}\right) \subseteq \widetilde{E}\left(a_{j}, \Omega, f_{2}\right)$ for all $1 \leq j \leq l$ and

$$
\liminf _{r \rightarrow+\infty} \frac{\sum_{j=1}^{l} \tilde{N}_{0}\left(r, \frac{1}{f_{1}-a_{j}}\right)}{\sum_{j=1}^{l} \widetilde{N}_{0}\left(r, \frac{1}{f_{2}-a_{j}}\right)}>\frac{1}{l-3},
$$

where $\widetilde{E}(a, \Omega, h)=\{z \mid h(z)-a=0, z \in \Omega\}$ for a meromorphic function $h(z)$ in $\Omega$, where each zero is counted only once, then $f_{1} \equiv f_{2}$. 
From Theorem 2.1, we can obtain the following corollary immediately.

Corollary 2.1 Let $f_{1}, f_{2}$ be two admissible meromorphic functions in $\Omega, a_{1}, a_{2}, \ldots, a_{l}$ be $l(\geq 5)$ distinct values. If $\widetilde{E}\left(a_{j}, \Omega, f_{1}\right) \subseteq \widetilde{E}\left(a_{j}, \Omega, f_{2}\right)$ for all $1 \leq j \leq l$ and $f_{1} \not \equiv f_{2}$, then

$$
\liminf _{r \rightarrow+\infty} \frac{\sum_{j=1}^{l} \tilde{N}_{0}\left(r, \frac{1}{f_{1}-a_{j}}\right)}{\sum_{j=1}^{l} \widetilde{N}_{0}\left(r, \frac{1}{f_{2}-a_{j}}\right)} \leq \frac{1}{l-3} .
$$

Remark 2.1 When $l=5$ and $\widetilde{E}\left(a_{j}, \Omega, f_{1}\right)=\widetilde{E}\left(a_{j}, \Omega, f_{2}\right)$ for all $1 \leq j \leq l$, in this case,

$$
\liminf _{r \rightarrow+\infty} \frac{\sum_{j=1}^{5} \tilde{N}_{0}\left(r, \frac{1}{f_{1}-a_{j}}\right)}{\sum_{j=1}^{5} \widetilde{N}_{0}\left(r, \frac{1}{f_{2}-a_{j}}\right)}=1>\frac{1}{2} .
$$

Then it follows $f_{1} \equiv f_{2}$ by Theorem 2.1. Thus, this shows that Theorem 2.1 is an improvement of Theorem $\mathrm{C}$.

Inspired by Question A, Theorem B, and Theorem 2.1, the second purpose of this paper is to investigate the uniqueness of meromorphic functions concerning small functions, and we obtain an analog of Nevanlinna's five-value theorem for meromorphic functions in a k-punctured complex plane.

Theorem 2.2 Let $f_{1}, f_{2}$ be two admissible meromorphic functions in $\Omega, \alpha_{1}, \alpha_{2}, \ldots, \alpha_{l}$ be $l(\geq$ 5) distinct small functions with respect to $f_{1}, f_{2}$. If $\widetilde{E}\left(\alpha_{j}, \Omega, f_{1}\right) \subseteq \widetilde{E}\left(\alpha_{j}, \Omega, f_{2}\right)$ for all $1 \leq j \leq l$ and

$$
\liminf _{r \rightarrow+\infty} \frac{\sum_{j=1}^{l} \tilde{N}_{0}\left(r, \frac{1}{f_{1}-\alpha_{j}}\right)}{\sum_{j=1}^{l} \widetilde{N}_{0}\left(r, \frac{1}{f_{2}-\alpha_{j}}\right)}>\frac{5}{2 l-5},
$$

where $\widetilde{E}(\alpha, \Omega, h)=\{z \mid h(z)-\alpha(z)=0, z \in \Omega\}$ for a meromorphic function $h(z)$ in $\Omega$, where each zero is counted only once, then $f_{1} \equiv f_{2}$.

Remark 2.2 Let $f$ be a nonconstant meromorphic function in a $k$-punctured plane $\Omega$, we denote by $S(f)$ a set of meromorphic function $a(z)$ in a $k$-punctured plane $\Omega$ satisfying $T_{0}(r, a)=S(r, f)$, and such a meromorphic function $a(z)$ in a $k$-punctured plane $\Omega$ is called a small function with respect to $f$.

From Theorem 2.2, the following results can be obtained immediately.

Corollary 2.2 Let $f_{1}, f_{2}$ be two admissible meromorphic functions in $\Omega$, and let $\alpha_{1}, \alpha_{2}, \ldots, \alpha_{l}$ be $l(\geq 5)$ distinct small functions with respect to $f_{1}, f_{2}$. If $\widetilde{E}\left(\alpha_{j}, \Omega, f_{1}\right) \subseteq \widetilde{E}\left(\alpha_{j}, \Omega, f_{2}\right)$ for all $1 \leq j \leq l$ and $f_{1} \not \equiv f_{2}$, then

$$
\liminf _{r \rightarrow+\infty} \frac{\sum_{j=1}^{l} \tilde{N}_{0}\left(r, \frac{1}{f_{1}-\alpha_{j}}\right)}{\sum_{j=1}^{l} \tilde{N}_{0}\left(r, \frac{1}{f_{2}-\alpha_{j}}\right)} \leq \frac{5}{2 l-5} .
$$


Corollary 2.3 Let $_{1}, f_{2}$ be two admissible meromorphic functions in $\Omega$, and let $\alpha_{1}, \alpha_{2}, \ldots, \alpha_{6}$ be six distinct small functions with respect to $f_{1}, f_{2}$. If $\widetilde{E}\left(\alpha_{j}, \Omega, f_{1}\right)=\widetilde{E}\left(\alpha_{j}, \Omega, f_{2}\right)$ for all $1 \leq j \leq 6$, then $f_{1} \equiv f_{2}$.

\section{The proof of Theorem 2.1}

To prove Theorem 2.1, we require the following lemmas.

Lemma 3.1 (see [35, Theorem 3]) Let $f, f_{1}, f_{2}$ be meromorphic functions in a k-punctured plane $\Omega$. Then:

(i) the function $T_{0}(r, f)$ is nonnegative, continuous, nondecreasing, and convex with respect to $\log r$ on $\left[r_{0},+\infty\right), T_{0}\left(r_{0}, f\right)=0$;

(ii) iff identically equals a constant, then $T_{0}(r, f)$ vanishes identically;

(iii) iff is not identically equal to zero, then $T_{0}(r, f)=T_{0}(r, 1 / f), r_{0} \leq r<+\infty$;

(iv) $T_{0}\left(r, f_{1} f_{2}\right) \leq T_{0}\left(r, f_{1}\right)+T_{0}\left(r, f_{2}\right)+O(1)$ and $T_{0}\left(r, f_{1}+f_{2}\right) \leq T_{0}\left(r, f_{1}\right)+T_{0}\left(r, f_{2}\right)+O(1)$ for $r_{0} \leq r<+\infty$;

(v) $T_{0}\left(r, \frac{1}{f-a}\right)=T_{0}(r, f)+O(1)$ for any fixed $a \in \mathbb{C}$.

By using Lemma 6 in [35], we can get the following lemma easily.

Lemma 3.2 Let $f$ be a nonconstant meromorphic function in a k-punctured plane $\Omega$ and $p$ be a positive integer, then

$$
m_{0}\left(r, \frac{f^{(p)}}{f}\right)=O\left(\log T_{0}(r, f)\right)+O\left(\log ^{+} r\right):=S(r, f), \quad r \rightarrow+\infty
$$

outside a set E of finite linear measure.

Remark 3.1 Obviously, if $f$ is admissible in a $k$-punctured plane $\Omega$, then

$$
m_{0}\left(r, \frac{f^{(p)}}{f}\right)=S(r, f)=o\left(T_{0}(r, f)\right)
$$

Lemma 3.3 ([34, Theorem 2.5]) Let $f$ be a nonconstant meromorphic function in a $k$ punctured plane $\Omega$, and let $a_{1}, a_{2}, \ldots, a_{q}(q \geq 3)$ be distinct complex numbers in the extended complex plane $\widehat{\mathbb{C}}:=\mathbb{C} \cup\{\infty\}$. Then, for $r_{0} \leq r<+\infty$,

$$
(q-2) T_{0}(r, f) \leq \sum_{\nu=1}^{q} \tilde{N}_{0}\left(r, \frac{1}{f-a_{v}}\right)+S(r, f),
$$

where $\tilde{n}_{0}\left(r, \frac{1}{f-a}\right)$ is the counting function of zeros off $-a$ in $\bar{\Omega}_{r}$ with the multiplicities reduced by 1 ,

$$
\widetilde{N}_{0}\left(r, \frac{1}{f-a_{v}}\right)=\int_{r_{0}}^{r} \frac{\widetilde{n}_{0}\left(t, \frac{1}{f-a_{v}}\right)}{t} d t
$$

$r \geq r_{0}$ and $S(r, f)$ is stated as in Lemma 3.2. 
Proof of Theorem 2.1 Without loss of generality, assume that $a_{j}(j=1,2, \ldots, l)$ are finite. In view of 3.3, it follows

$$
(l-2) T_{0}\left(r, f_{1}\right) \leq \sum_{j=1}^{l} \tilde{N}_{0}\left(r, \frac{1}{f_{1}-a_{j}}\right)+S\left(r, f_{1}\right)
$$

and

$$
(l-2) T_{0}\left(r, f_{2}\right) \leq \sum_{j=1}^{l} \widetilde{N}_{0}\left(r, \frac{1}{f_{2}-a_{j}}\right)+S\left(r, f_{2}\right) .
$$

Suppose that $f_{1} \not \equiv f_{2}$. In view of $\widetilde{E}\left(a_{j}, \Omega, f_{1}\right) \subseteq \widetilde{E}\left(a_{j}, \Omega, f_{2}\right)$ for all $1 \leq j \leq l$, then it yields

$$
\sum_{j=1}^{l} \widetilde{N}_{0}\left(r, \frac{1}{f_{1}-a_{j}}\right) \leq \widetilde{N}_{0}\left(r, \frac{1}{f_{1}-f_{2}}\right) \leq T_{0}\left(r, f_{1}\right)+T_{0}\left(r, f_{2}\right)+O(1) .
$$

Thus, we have

$$
\begin{aligned}
& \sum_{j=1}^{l} \widetilde{N}_{0}\left(r, \frac{1}{f_{1}-a_{j}}\right) \\
& \quad \leq\left(\frac{1}{l-2}+o(1)\right) \sum_{j=1}^{l} \widetilde{N}_{0}\left(r, \frac{1}{f_{1}-a_{j}}\right)+\left(\frac{1}{l-2}+o(1)\right) \sum_{j=1}^{l} \tilde{N}_{0}\left(r, \frac{1}{f_{2}-a_{j}}\right)
\end{aligned}
$$

for all $r \notin E$, which implies

$$
\left(\frac{l-3}{l-2}+o(1)\right) \sum_{j=1}^{l} \tilde{N}_{0}\left(r, \frac{1}{f_{1}-a_{j}}\right) \leq\left(\frac{1}{l-2}+o(1)\right) \sum_{j=1}^{l} \tilde{N}_{0}\left(r, \frac{1}{f_{2}-a_{j}}\right)
$$

for all $r \notin E$. Hence, it follows

$$
\liminf _{r \rightarrow+\infty} \frac{\sum_{j=1}^{l} \widetilde{N}_{0}\left(r, \frac{1}{f_{1}-\alpha_{j}}\right)}{\sum_{j=1}^{l} \widetilde{N}_{0}\left(r, \frac{1}{f_{2}-\alpha_{j}}\right)} \leq \frac{1}{l-3}
$$

which is a contradiction. Thus, $f_{1} \equiv f_{2}$.

Therefore, this completes the proof of Theorem 2.1.

\section{The proof of Theorem $\mathbf{2 . 2}$}

To prove Theorem 2.2, we will require the following lemmas.

Lemma 4.1 Let $f_{1}(z)$ and $f_{2}(z)$ be two admissible meromorphic functions in a k-punctured plane $\Omega, a_{t}(z)(\not \equiv 0,1) \in S(r):=S\left(f_{1}\right) \cap S\left(f_{2}\right), t=1,2$, be not equal to constants simultaneously, and let

$$
F_{s}(z)=\left|\begin{array}{ccc}
f_{s} f_{s}^{\prime} & f_{s}^{\prime} & f_{s}^{2}-f_{s} \\
a_{1} a_{1}^{\prime} & a_{1}^{\prime} & a_{1}^{2}-a_{1} \\
a_{2} a_{2}^{\prime} & a_{2}^{\prime} & a_{2}^{2}-a_{2}
\end{array}\right| \quad \text { for } s=1,2
$$


Then:

(i) $F_{s}(z) \not \equiv 0$ for $s=1,2$.

(ii)

$$
\begin{aligned}
2 T_{0}\left(r, f_{s}\right)< & \widetilde{N}_{0}\left(r, \frac{1}{f_{s}-1}\right)+\widetilde{N}_{0}\left(r, \frac{1}{f_{s}}\right)+\widetilde{N}_{0}\left(r, f_{s}\right)+\widetilde{N}_{0}\left(r, \frac{1}{f_{s}-a_{1}}\right) \\
& +\widetilde{N}_{0}\left(r, \frac{1}{f_{s}-a_{2}}\right)+S\left(r, f_{1}\right)+S\left(r, f_{2}\right) \quad \text { for } s=1,2 .
\end{aligned}
$$

Proof (i) Assume that $F_{1}(z) \equiv 0$. Thus, we can rewrite (4.1) as the following form:

$$
\left(\frac{a_{1}^{\prime}}{a_{1}}-\frac{a_{2}^{\prime}}{a_{2}}\right)\left(\frac{f_{1}^{\prime}}{f_{1}-1}-\frac{a_{2}^{\prime}}{a_{2}-1}\right)-\left(\frac{a_{1}^{\prime}}{a_{1}-1}-\frac{a_{2}^{\prime}}{a_{2}-1}\right)\left(\frac{f_{1}^{\prime}}{f_{1}}-\frac{a_{2}^{\prime}}{a_{2}}\right) \equiv 0 .
$$

Next, we will divide the proof into four cases as follows.

Case 1. If $\frac{a_{1}^{\prime}}{a_{1}} \equiv \frac{a_{2}^{\prime}}{a_{2}}$, then it follows $a_{1}=\eta_{1} a_{2}$, where $\eta_{1} \neq 1$ is a constant. From the assumptions of this lemma, it yields $\frac{a_{1}^{\prime}}{a_{1}-1} \not \equiv \frac{a_{2}^{\prime}}{a_{2}-1}$, which implies $\frac{f_{1}^{\prime}}{f_{1}} \equiv \frac{a_{2}^{\prime}}{a_{2}}$. Thus, we have $f_{1}(z)=\eta_{2} a_{2}(z)$, where $\eta_{2}$ is a constant. Therefore, we get a contradiction.

Case 2. If $\frac{a_{1}^{\prime}}{a_{1}-1} \equiv \frac{a_{2}^{\prime}}{a_{2}-1}$. By using the same argument as in Case 1 , we also get a contradiction.

Case 3. If $\frac{a_{1}^{\prime}}{a_{1}} \not \equiv \frac{a_{2}^{\prime}}{a_{2}}, \frac{a_{1}^{\prime}}{a_{1}-1} \not \equiv \frac{a_{2}^{\prime}}{a_{2}-1}$ and $\frac{a_{1}^{\prime}}{a_{1}}-\frac{a_{2}^{\prime}}{a_{2}} \equiv \frac{a_{1}^{\prime}}{a_{1}-1}-\frac{a_{2}^{\prime}}{a_{2}-1}$. Thus it follows from (4.1) that

$$
\frac{f_{1}^{\prime}}{f_{1}-1}-\frac{f_{1}^{\prime}}{f_{1}} \equiv \frac{a_{2}^{\prime}}{a_{2}-1}-\frac{a_{2}^{\prime}}{a_{2}}
$$

By a simple integral, we have $\frac{1}{f_{1}}=1-\eta_{3}\left(1-\frac{1}{a_{2}}\right)$, where $\eta_{3}$ is a constant, a contradiction.

Case 4. If $\frac{a_{1}^{\prime}}{a_{1}} \not \equiv \frac{a_{2}^{\prime}}{a_{2}}, \frac{a_{1}^{\prime}}{a_{1}-1} \not \equiv \frac{a_{2}^{\prime}}{a_{2}-1}$ and $\frac{a_{1}^{\prime}}{a_{1}}-\frac{a_{2}^{\prime}}{a_{2}} \not \equiv \frac{a_{1}^{\prime}}{a_{1}-1}-\frac{a_{2}^{\prime}}{a_{2}-1}$. Thus, we can rewrite (4.2) as the following form:

$$
\left(\frac{a_{1}^{\prime}}{a_{1}}-\frac{a_{2}^{\prime}}{a_{2}}\right) \frac{f_{1}^{\prime}}{f_{1}-1}-\left(\frac{a_{1}^{\prime}}{a_{1}-1}-\frac{a_{2}^{\prime}}{a_{2}-1}\right) \frac{f_{1}^{\prime}}{f_{1}} \equiv \frac{a_{1}^{\prime}}{a_{1}} \frac{a_{2}^{\prime}}{a_{2}-1}-\frac{a_{2}^{\prime}}{a_{2}} \frac{a_{1}^{\prime}}{a_{1}-1} .
$$

By observing (4.3), the zeros of $f_{1}-1$ in $\Omega$ can only occur at the zeros, 1-points and poles of $a_{1}(z)$ and $a_{2}(z)$, and the zeros of $\frac{a_{1}^{\prime}}{a_{1}}-\frac{a_{2}^{\prime}}{a_{2}}$ in $\Omega$. Thus, we have

$$
\begin{aligned}
\widetilde{N}_{0}\left(r, \frac{1}{f_{1}-1}\right) \leq & \sum_{j=1}^{2}\left\{N_{0}\left(r, a_{j}\right)+N_{0}\left(r, \frac{1}{a_{j}}\right)+N_{0}\left(r, \frac{1}{a_{j}-1}\right)\right\} \\
& +N_{0}\left(r, \frac{1}{\frac{a_{1}^{\prime}}{a_{1}}-\frac{a_{2}^{\prime}}{a_{2}}}\right) \\
= & S\left(r, f_{1}\right)+S\left(r, f_{2}\right) .
\end{aligned}
$$

Similarly, we have

$$
\tilde{N}_{0}\left(r, \frac{1}{f_{1}}\right)=S\left(r, f_{1}\right)+S\left(r, f_{2}\right)
$$


Further, the poles of $f_{1}$ in $\Omega$ can only occur at the zeros, 1-points and poles of $a_{1}(z)$ and $a_{2}(z)$, and the zeros of $\left(\frac{a_{1}^{\prime}}{a_{1}}-\frac{a_{2}^{\prime}}{a_{2}}\right)-\left(\frac{a_{1}^{\prime}}{a_{1}-1}-\frac{a_{2}^{\prime}}{a_{2}-1}\right)$ in $\Omega$. By a simple calculation, we have

$$
\tilde{N}_{0}\left(r, f_{1}\right)=S\left(r, f_{1}\right)+S\left(r, f_{2}\right) .
$$

By Lemma 3.3 and from (4.4)-(4.6), it follows

$$
\begin{aligned}
T_{0}\left(r, f_{1}\right) & <\widetilde{N}_{0}\left(r, \frac{1}{f_{1}-1}\right)+\widetilde{N}_{0}\left(r, \frac{1}{f_{1}}\right)+\widetilde{N}_{0}\left(r, f_{1}\right)+S\left(r, f_{1}\right) \\
& =S\left(r, f_{1}\right)+S\left(r, f_{2}\right),
\end{aligned}
$$

a contradiction.

If $F_{2}(z) \equiv 0$, by using the same argument as above, we also get a contradiction. Then we prove (i).

(ii) Let

$$
\begin{aligned}
& \delta(z)=\frac{1}{3} \min \left\{1,\left|a_{1}(z)\right|,\left|a_{2}(z)\right|,\left|a_{1}(z)-1\right|,\left|a_{2}(z)-1\right|,\left|a_{1}(z)-a_{2}(z)\right|, z \in \Omega\right\}, \\
& \theta_{t}(r)=\left\{\theta:\left|f_{1}\left(r e^{i \theta}\right)-a_{t}\left(r^{i \theta}\right)\right| \leq \delta\left(r^{i \theta}\right)\right\} \quad(t=1,2), \\
& \theta_{3}(r)=\left\{\theta:\left|f_{1}\left(r e^{i \theta}\right)\right| \leq \delta\left(r^{i \theta}\right)\right\}, \\
& \theta_{4}(r)=\left\{\theta:\left|f_{1}\left(r e^{i \theta}\right)-1\right| \leq \delta\left(r^{i \theta}\right)\right\} .
\end{aligned}
$$

Then it follows

$$
\begin{aligned}
\frac{1}{2 \pi} & \int_{0}^{2 \pi} \log \frac{1}{\delta\left(r e^{i \theta}\right)} d \theta \\
\leq & \frac{1}{2 \pi} \int_{0}^{2 \pi} \log \max \left\{1, \frac{1}{\left|a_{1}(z)\right|}, \frac{1}{\left|a_{2}(z)\right|}, \frac{1}{\left|a_{1}(z)-1\right|}, \frac{1}{\left|a_{2}(z)-1\right|},\right. \\
& \left.\frac{1}{\left|a_{1}(z)-a_{2}(z)\right|}, z \in \Omega\right\} d \theta+\log 3 \\
\leq & m\left(r, \frac{1}{a_{1}}\right)+m\left(r, \frac{1}{a_{2}}\right)+m\left(r, \frac{1}{a_{1}-1}\right)+m\left(r, \frac{1}{a_{2}-1}\right) \\
& +m\left(r, \frac{1}{a_{1}-a_{2}}\right)+4 \log 2 .
\end{aligned}
$$

Similarly, for any $c_{j}, j=1,2, \ldots, k$, we have

$$
\begin{aligned}
\frac{1}{2 \pi} & \int_{0}^{2 \pi} \log \frac{1}{\delta\left(c_{j}+\frac{1}{r} e^{i \theta}\right)} d \theta \\
\leq & \frac{1}{2 \pi} \int_{0}^{2 \pi} \log \max \left\{1, \frac{1}{\left|a_{1}\left(c_{j}+\frac{1}{r} e^{i \theta}\right)\right|}, \frac{1}{\left|a_{2}\left(c_{j}+\frac{1}{r} e^{i \theta}\right)\right|}, \frac{1}{\left|a_{1}\left(c_{j}+\frac{1}{r} e^{i \theta}\right)-1\right|},\right. \\
& \left.\frac{1}{\left|a_{2}\left(c_{j}+\frac{1}{r} e^{i \theta}\right)-1\right|}, \frac{1}{\left|a_{1}\left(c_{j}+\frac{1}{r} e^{i \theta}\right)-a_{2}\left(c_{j}+\frac{1}{r} e^{i \theta}\right)\right|}, z \in \Omega\right\} d \theta+\log 3 \\
\leq & m\left(\frac{1}{r}, \frac{1}{a_{1}\left(c_{j}+z\right)}\right)+m\left(r, \frac{1}{a_{2}\left(c_{j}+z\right)}\right)+m\left(r, \frac{1}{a_{1}\left(c_{j}+z\right)-1}\right)
\end{aligned}
$$




$$
+m\left(r, \frac{1}{a_{2}\left(c_{j}+z\right)-1}\right)+m\left(r, \frac{1}{a_{1}\left(c_{j}+z\right)-a_{2}\left(c_{j}+z\right)}\right)+4 \log 2 .
$$

Further, for $j=1,2, \ldots, k$,

$$
\frac{1}{2 \pi} \int_{0}^{2 \pi} \log \frac{1}{\delta\left(r_{0} e^{i \theta}\right)} d \theta=O(1), \quad \frac{1}{2 \pi} \int_{0}^{2 \pi} \log \frac{1}{\delta\left(c_{j}+\frac{1}{r_{0}} e^{i \theta}\right)} d \theta=O(1) .
$$

Thus, it follows

$$
\begin{aligned}
\frac{1}{2 \pi} \int_{0}^{2 \pi} \log \frac{1}{\delta\left(r e^{i \theta}\right)} d \theta+\sum_{j=1}^{k} \frac{1}{2 \pi} \int_{0}^{2 \pi} \log \frac{1}{\delta\left(c_{j}+\frac{1}{r} e^{i \theta}\right)} d \theta \\
\quad-\frac{1}{2 \pi} \int_{0}^{2 \pi} \log \frac{1}{\delta\left(r_{0} e^{i \theta}\right)} d \theta-\sum_{j=1}^{k} \frac{1}{2 \pi} \int_{0}^{2 \pi} \log \frac{1}{\delta\left(c_{j}+\frac{1}{r_{0}} e^{i \theta}\right)} d \theta \\
\leq m_{0}\left(r, \frac{1}{a_{1}}\right)+m_{0}\left(r, \frac{1}{a_{2}}\right)+m_{0}\left(r, \frac{1}{a_{1}-1}\right) \\
\quad+m_{0}\left(r, \frac{1}{a_{2}-1}\right)+m\left(r, \frac{1}{a_{1}-a_{2}}\right)+O(1) \\
\leq S\left(r, f_{1}\right)+S\left(r, f_{2}\right) .
\end{aligned}
$$

On the other hand, taking

$$
\begin{aligned}
& f_{1} f_{1}^{\prime}=\left(f_{1}-a_{1}\right)\left(f_{1}^{\prime}-a_{1}^{\prime}\right)+a_{1}^{\prime}\left(f_{1}-a_{1}\right)+a_{1}\left(f_{1}^{\prime}-a_{1}^{\prime}\right)+a_{1} a_{2}^{\prime}:=F_{1}, \\
& f_{1}^{\prime}=\left(f_{1}^{\prime}-a_{1}^{\prime}\right)+a_{1}^{\prime}:=F_{2}, \\
& f_{1}^{2}-f_{1}=\left(f_{1}-a_{1}\right)^{2}+\left(2 a_{1}-1\right)\left(f_{1}-a_{1}\right)+a_{1}^{2}-a_{1}:=F_{3},
\end{aligned}
$$

and substituting these into (4.22), by a simple calculation, we have

$$
F=\left|\begin{array}{ccc}
F_{1}-a_{1} a_{2}^{\prime} & F_{2}-a_{1}^{\prime} & F_{3}-a_{1}^{2}+a_{1} \\
a_{1} a_{1}^{\prime} & a_{1}^{\prime} & a_{1}^{2}-a_{1} \\
a_{2} a_{2}^{\prime} & a_{2}^{\prime} & a_{2}^{2}-a_{2}
\end{array}\right| .
$$

From the definition of $\theta_{1}(r)$ and $\delta(z)$, we have

$$
\left|f_{1}\left(r e^{i \theta}\right)-a_{1}\left(r e^{i \theta}\right)\right| \leq \delta\left(r e^{i \theta}\right) \leq 1+\left|a_{1}\left(r e^{i \theta}\right)\right| \quad \text { as } \theta \in \theta_{1}(r)
$$

and

$$
\begin{aligned}
& \frac{1}{2 \pi} \int_{\theta_{1}(r)} \log ^{+}\left|\frac{F}{f_{1}-a_{1}}\right| d \theta \\
& \quad \leq m\left(r, \frac{f_{1}^{\prime}-a_{1}^{\prime}}{f_{1}-a_{1}}\right)+O\left(m\left(r, a_{1}\right)+m\left(r, a_{2}\right)+m\left(r, a_{1}^{\prime}\right)+m\left(r, a_{2}^{\prime}\right)\right) \\
& \quad<S\left(r, f_{1}\right)+S\left(r, f_{2}\right) .
\end{aligned}
$$


On the other hand, we have $\left|f_{1}\left(r e^{i \theta}\right)-a_{1}\left(r e^{i \theta}\right)\right| \geq \delta\left(r e^{i \theta}\right)$ as $\theta \notin \theta_{1}(r)$, that is,

$$
\frac{1}{\left|f_{1}\left(r e^{i \theta}\right)-a_{1}\left(r e^{i \theta}\right)\right|} \leq \frac{1}{\delta\left(r e^{i \theta}\right)} \quad \text { as } \theta \notin \theta_{1}(r)
$$

By combining (4.10) and (4.11), we have

$$
\begin{aligned}
& m\left(r, \frac{1}{f_{1}-a_{1}}\right) \\
& \leq \frac{1}{2 \pi} \int_{\theta_{1}(r)} \log ^{+}\left|\frac{F}{f_{1}-a_{1}}\right| d \theta+\frac{1}{2 \pi} \int_{\theta_{1}(r)} \log ^{+}\left|\frac{1}{F}\right| d \theta \\
&+\frac{1}{2 \pi} \int_{[0,2 \pi]-\theta_{1}(r)} \log \left|\frac{1}{\delta}\right| d \theta \\
& \leq \frac{1}{2 \pi} \int_{\theta_{1}(r)} \log ^{+}\left|\frac{1}{F\left(r e^{i \theta}\right)}\right| d \theta+\frac{1}{2 \pi} \int_{0}^{2 \pi} \log \left|\frac{1}{\delta\left(r e^{i \theta}\right)}\right| d \theta \\
&+S\left(r, f_{1}\right)+S\left(r, f_{2}\right) .
\end{aligned}
$$

Similarly, we have

$$
\begin{aligned}
& m\left(\frac{1}{r}, \frac{1}{f_{1}\left(c_{j}+\frac{1}{r} e^{i \theta}\right)-a_{1}\left(c_{j}+\frac{1}{r} e^{i \theta}\right)}\right) \\
& \leq \frac{1}{2 \pi} \int_{\theta_{1}(r)} \log ^{+}\left|\frac{F\left(c_{j}+\frac{1}{r} e^{i \theta}\right)}{f_{1}\left(c_{j}+\frac{1}{r} e^{i \theta}\right)-a_{1}\left(c_{j}+\frac{1}{r} e^{i \theta}\right)}\right| d \theta \\
&+\frac{1}{2 \pi} \int_{\theta_{1}(r)} \log ^{+}\left|\frac{1}{F\left(c_{j}+\frac{1}{r} e^{i \theta}\right)}\right| d \theta+\frac{1}{2 \pi} \int_{[0,2 \pi]-\theta_{1}(r)} \log \left|\frac{1}{\delta\left(c_{j}+\frac{1}{r} e^{i \theta}\right)}\right| d \theta \\
& \leq \frac{1}{2 \pi} \int_{\theta_{1}(r)} \log ^{+}\left|\frac{1}{F\left(c_{j}+\frac{1}{r} e^{i \theta}\right)}\right| d \theta+\frac{1}{2 \pi} \int_{0}^{2 \pi} \log \left|\frac{1}{\delta\left(c_{j}+\frac{1}{r} e^{i \theta}\right)}\right| d \theta \\
&+S\left(r, f_{1}\right)+S\left(r, f_{2}\right) .
\end{aligned}
$$

Since

$$
\begin{aligned}
& m\left(r_{0}, \frac{1}{f_{1}-a_{1}}\right)=O(1), \quad \frac{1}{2 \pi} \int_{\theta_{1}\left(r_{0}\right)} \log ^{+}\left|\frac{1}{F\left(r_{0} e^{i \theta}\right)}\right| d \theta=O(1), \\
& m\left(\frac{1}{r_{0}}, \frac{1}{f_{1}\left(c_{j}+\frac{1}{r_{0}} e^{i \theta}\right)-a_{1}\left(c_{j}+\frac{1}{r_{0}} e^{i \theta}\right)}\right)=O(1)
\end{aligned}
$$

and

$$
\frac{1}{2 \pi} \int_{\theta_{1}\left(r_{0}\right)} \log ^{+}\left|\frac{1}{F\left(c_{j}+\frac{1}{r_{0}} e^{i \theta}\right)}\right| d \theta=O(1), \quad j=1,2, \ldots, k
$$

by combining (4.7), (4.12), and (4.13), it follows

$$
m_{0}\left(r, \frac{1}{f_{1}-a_{1}}\right)
$$


Xu et al. Journal of Inequalities and Applications

(2019) 2019:289

Page 11 of 15

$$
\begin{aligned}
\leq & \frac{1}{2 \pi} \int_{\theta_{1}(r)} \log ^{+}\left|\frac{1}{F\left(r e^{i \theta}\right)}\right| d \theta+\sum_{j=1}^{k} \frac{1}{2 \pi} \int_{\theta_{1}(r)} \log ^{+}\left|\frac{1}{F\left(c_{j}+\frac{1}{r} e^{i \theta}\right)}\right| d \theta \\
& -\frac{1}{2 \pi} \int_{\theta_{1}\left(r_{0}\right)} \log ^{+}\left|\frac{1}{F\left(r_{0} e^{i \theta}\right)}\right| d \theta-\sum_{j=1}^{k} \frac{1}{2 \pi} \int_{\theta_{1}\left(r_{0}\right)} \log ^{+}\left|\frac{1}{F\left(c_{j}+\frac{1}{r_{0}} e^{i \theta}\right)}\right| d \theta \\
& +S\left(r, f_{1}\right)+S\left(r, f_{2}\right) .
\end{aligned}
$$

By using the same argument as above, we have

$$
\begin{aligned}
m_{0}( & \left.r, \frac{1}{f_{1}-a_{2}}\right) \\
\leq & \frac{1}{2 \pi} \int_{\theta_{2}(r)} \log ^{+}\left|\frac{1}{F\left(r e^{i \theta}\right)}\right| d \theta+\sum_{j=1}^{k} \frac{1}{2 \pi} \int_{\theta_{2}(r)} \log ^{+}\left|\frac{1}{F\left(c_{j}+\frac{1}{r} e^{i \theta}\right)}\right| d \theta \\
& -\frac{1}{2 \pi} \int_{\theta_{2}\left(r_{0}\right)} \log ^{+}\left|\frac{1}{F\left(r_{0} e^{i \theta}\right)}\right| d \theta-\sum_{j=1}^{k} \frac{1}{2 \pi} \int_{\theta_{2}\left(r_{0}\right)} \log ^{+}\left|\frac{1}{F\left(c_{j}+\frac{1}{r_{0}} e^{i \theta}\right)}\right| d \theta \\
& +S\left(r, f_{1}\right)+S\left(r, f_{2}\right) ; \\
m_{0}\left(r, \frac{1}{f_{1}}\right) & \frac{1}{2 \pi} \int_{\theta_{3}(r)} \log ^{+}\left|\frac{1}{F\left(r e^{i \theta}\right)}\right| d \theta+\sum_{j=1}^{k} \frac{1}{2 \pi} \int_{\theta_{3}(r)} \log ^{+}\left|\frac{1}{F\left(c_{j}+\frac{1}{r} e^{i \theta}\right)}\right| d \theta \\
\quad & \frac{1}{2 \pi} \int_{\theta_{3}\left(r_{0}\right)} \log ^{+}\left|\frac{1}{F\left(r_{0} e^{i \theta}\right)}\right| d \theta-\sum_{j=1}^{k} \frac{1}{2 \pi} \int_{\theta_{3}\left(r_{0}\right)} \log ^{+}\left|\frac{1}{F\left(c_{j}+\frac{1}{r_{0}} e^{i \theta}\right)}\right| d \theta \\
& +S\left(r, f_{1}\right)+S\left(r, f_{2}\right) ;
\end{aligned}
$$

and

$$
\begin{aligned}
& m_{0}\left(r, \frac{1}{f_{1}-1}\right) \\
& \leq \frac{1}{2 \pi} \int_{\theta_{4}(r)} \log ^{+}\left|\frac{1}{F\left(r e^{i \theta}\right)}\right| d \theta+\sum_{j=1}^{k} \frac{1}{2 \pi} \int_{\theta_{4}(r)} \log ^{+}\left|\frac{1}{F\left(c_{j}+\frac{1}{r} e^{i \theta}\right)}\right| d \theta \\
&-\frac{1}{2 \pi} \int_{\theta_{4}\left(r_{0}\right)} \log ^{+}\left|\frac{1}{F\left(r_{0} e^{i \theta}\right)}\right| d \theta-\sum_{j=1}^{k} \frac{1}{2 \pi} \int_{\theta_{4}\left(r_{0}\right)} \log ^{+}\left|\frac{1}{F\left(c_{j}+\frac{1}{r_{0}} e^{i \theta}\right)}\right| d \theta \\
&+S\left(r, f_{1}\right)+S\left(r, f_{2}\right) .
\end{aligned}
$$

Since $\theta(r) \in[0,2 \pi)$, then from (4.14)-(4.17) it yields

$$
\begin{aligned}
& m_{0}\left(r, \frac{1}{f_{1}-a_{1}}\right)+m_{0}\left(r, \frac{1}{f_{1}-a_{2}}\right)+m_{0}\left(r, \frac{1}{f_{1}}\right)+m_{0}\left(r, \frac{1}{f_{1}-1}\right) \\
& \quad<m_{0}\left(r, \frac{1}{F}\right)+S\left(r, f_{1}\right)+S\left(r, f_{2}\right) .
\end{aligned}
$$


If $z_{0}$ is a zero of $f_{1}$ or $f_{1}-1$ or $f_{1}-a_{1}$ or $f_{1}-a_{2}$ in $\Omega$ of multiplies $p>1$ and not a pole of $a_{1}$ or $a_{2}$ in $\Omega$, then $z_{0}$ must be a zero of $F_{1}(z)$ in $\Omega$ of multiplies $p-1$. Thus, it follows

$$
\begin{aligned}
4 T_{0}\left(r, f_{1}\right)< & N_{0}\left(r, \frac{1}{f_{1}}\right)+N_{0}\left(r, \frac{1}{f_{1}-1}\right)+N_{0}\left(r, \frac{1}{f_{1}-a_{1}}\right)+N_{0}\left(r, \frac{1}{f_{1}-a_{2}}\right) \\
& -N_{0}\left(r, \frac{1}{F_{1}}\right)+T_{0}\left(r, F_{1}\right)+O(1)+S\left(r, f_{1}\right)+S\left(r, f_{2}\right) \\
< & \widetilde{N}_{0}\left(r, \frac{1}{f_{1}}\right)+\widetilde{N}_{0}\left(r, \frac{1}{f_{1}-1}\right)+\widetilde{N}_{0}\left(r, \frac{1}{f_{1}-a_{1}}\right)+\widetilde{N}_{0}\left(r, \frac{1}{f_{1}-a_{2}}\right) \\
& +T_{0}\left(r, F_{1}\right)+O(1)+S\left(r, f_{1}\right)+S\left(r, f_{2}\right) .
\end{aligned}
$$

In addition, from the definition of $F_{1}(z)$, we can get

$$
\begin{aligned}
& m_{0}\left(r, F_{1}\right)<2 m_{0}\left(r, f_{1}\right)+S\left(r, f_{1}\right)+S\left(r, f_{2}\right), \\
& N_{0}\left(r, F_{1}\right)<2 N_{0}\left(r, f_{1}\right)+\widetilde{N}_{0}\left(r, f_{1}\right)+S\left(r, f_{1}\right)+S\left(r, f_{2}\right) .
\end{aligned}
$$

Hence, from (4.19)-(4.21), we can get Lemma 4.1(ii).

Therefore, this completes the proof of Lemma 4.1.

Lemma 4.2 Let $f_{1}(z)$ and $f_{2}(z)$ be two admissible meromorphic functions in a k-punctured plane $\Omega, \alpha_{j}(z)(\neq \equiv, 1) \in S\left(f_{1}\right) \cap S\left(f_{2}\right), j=1,2, \ldots, 5$, be five distinct meromorphic functions in a k-punctured plane $\Omega$, then

$$
2 T_{0}\left(r, f_{s}\right)<\sum_{j=1}^{5} \widetilde{N}_{0}\left(r, \frac{1}{f_{s}-\alpha_{j}}\right)+S\left(r, f_{1}\right)+S\left(r, f_{2}\right), \quad s=1,2 .
$$

Proof Set

$$
\begin{aligned}
& g_{s}=\frac{f_{s}-\alpha_{4}}{f_{s}-\alpha_{5}} \frac{\alpha_{3}-\alpha_{5}}{\alpha_{3}-\alpha_{4}} \quad(s=1,2), \\
& a_{j}=\frac{\alpha_{j}-\alpha_{4}}{\alpha_{j}-\alpha_{5}} \frac{\alpha_{3}-\alpha_{5}}{\alpha_{3}-\alpha_{4}} \quad(j=1,2) .
\end{aligned}
$$

Then it yields

$$
\begin{aligned}
& \left|T_{0}\left(r, g_{s}\right)-T_{0}\left(r, f_{s}\right)\right|<S\left(r, f_{1}\right)+S\left(r, f_{2}\right), \quad \text { for } s=1,2, \\
& S\left(r, f_{1}\right)+S\left(r, f_{2}\right)=S\left(r, g_{1}\right)+S\left(r, g_{2}\right) .
\end{aligned}
$$

Here we will consider three cases as follows.

Case 1. If $g_{1}$ and $g_{2}$ are admissible, then by applying Lemma 4.1 for $g_{1}, g_{2}, a_{1}, a_{2}$, we have

$$
\begin{aligned}
2 T_{0}\left(r, g_{s}\right)< & \widetilde{N}_{0}\left(r, g_{s}\right)+\widetilde{N}_{0}\left(r, \frac{1}{g_{s}}\right)+\tilde{N}_{0}\left(r, \frac{1}{g_{s}-1}\right)+\widetilde{N}_{0}\left(r, \frac{1}{g_{s}-a_{1}}\right) \\
& +\widetilde{N}_{0}\left(r, \frac{1}{g_{s}-a_{2}}\right)+S\left(r, g_{1}\right)+S\left(r, g_{2}\right)
\end{aligned}
$$


for $s=1$, 2. From the notations of $g_{1}$ and $g_{2}$ and $\alpha_{j} \in S\left(f_{1}\right) \cap S\left(f_{2}\right)$, we have

$$
\begin{aligned}
& \tilde{N}_{0}\left(r, g_{s}\right)<N_{0}\left(r, \frac{1}{f_{s}-\alpha_{5}}\right)+O\left(\sum_{j=1}^{5} T_{0}\left(r, \alpha_{j}\right)\right), \\
& \tilde{N}_{0}\left(r, \frac{1}{g_{s}}\right)<N_{0}\left(r, \frac{1}{f_{s}-\alpha_{4}}\right)+O\left(\sum_{j=1}^{5} T_{0}\left(r, \alpha_{j}\right)\right),
\end{aligned}
$$

and

$$
\widetilde{N}_{0}\left(r, \frac{1}{g_{s}-a_{j}}\right)<N_{0}\left(r, \frac{1}{f_{s}-\alpha_{j}}\right)+O\left(\sum_{j=1}^{5} T_{0}\left(r, \alpha_{j}\right)\right)
$$

for $s=1,2 ; j=1,2$. Substituting (4.26) -(4.28) into (4.25), we can get (4.22) easily.

Case 2. If $g_{1}$ and $g_{2}$ are rational, then from Remark 2.1 we have $T_{0}\left(r, g_{s}\right)=O(\log r)=$ $S\left(r, f_{1}\right)+S\left(r, f_{2}\right)$ for $s=1,2$. Thus, combining (4.23) and (4.24), it yields $T_{0}\left(r, f_{s}\right)=S\left(r, f_{1}\right)+$ $S\left(r, f_{2}\right)$ for $s=1,2$. Hence the conclusions hold.

Case 3. If one of $g_{1}, g_{2}$ is rational, without loss of generality assume that $g_{1}$ is rational and $g_{2}$ is admissible. From Case 2 and Case 1 , we have $T_{0}\left(r, f_{1}\right)=S\left(r, f_{1}\right)+S\left(r, f_{2}\right)$ and

$$
T_{0}\left(r, f_{2}\right)<\sum_{j=1}^{5} \widetilde{N}_{0}\left(r, \frac{1}{f_{2}-\alpha_{j}}\right)+S\left(r, f_{1}\right)+S\left(r, f_{2}\right) .
$$

Then the conclusion holds.

From Cases 1-3, this completes the proof of Lemma 4.2.

Proof of Theorem 2.2 Take any distinct $s_{1}, \ldots, s_{5} \in\{1,2, \ldots, l\}$, and in view of Lemma 4.2, it follows

$$
2 T_{0}\left(r, f_{i}\right)<\sum_{j=1}^{5} \widetilde{N}_{0}\left(r, \frac{1}{f_{i}-\alpha_{s_{j}}}\right)+S(r), \quad i=1,2 .
$$

Thus, we can conclude

$$
2\left(\begin{array}{l}
l \\
5
\end{array}\right) T_{0}\left(r, f_{i}\right) \leq \frac{5}{l}\left(\begin{array}{l}
l \\
5
\end{array}\right) \sum_{j=1}^{l} \widetilde{N}_{0}\left(r, \frac{1}{f_{i}-\alpha_{j}}\right)+S(r), \quad i=1,2,
$$

that is,

$$
T_{0}\left(r, f_{i}\right) \leq \frac{5}{2 l} \sum_{j=1}^{l} \widetilde{N}_{0}\left(r, \frac{1}{f_{i}-\alpha_{j}}\right)+S(r), \quad i=1,2 .
$$

Suppose that $f_{1} \not \equiv f_{2}$. In view of $\widetilde{E}\left(\alpha_{j}, \Omega, f_{1}\right) \subseteq \widetilde{E}\left(\alpha_{j}, \Omega, f_{2}\right)$ for all $1 \leq j \leq l$, we have

$$
\sum_{j=1}^{l} \widetilde{N}_{0}\left(r, \frac{1}{f_{1}-\alpha_{j}}\right) \leq \widetilde{N}_{0}\left(r, \frac{1}{f_{1}-f_{2}}\right) \leq T_{0}\left(r, f_{1}\right)+T_{0}\left(r, f_{2}\right)+O(1) .
$$


In view of (4.30) and (4.31), we can deduce

$$
\begin{aligned}
& \sum_{j=1}^{l} \tilde{N}_{0}\left(r, \frac{1}{f_{1}-\alpha_{j}}\right) \\
& \quad \leq\left(\frac{5}{2 l}+o(1)\right) \sum_{j=1}^{l} \tilde{N}_{0}\left(r, \frac{1}{f_{1}-\alpha_{j}}\right)+\left(\frac{5}{2 l}+o(1)\right) \sum_{j=1}^{l} \tilde{N}_{0}\left(r, \frac{1}{f_{2}-\alpha_{j}}\right)
\end{aligned}
$$

for $r \notin E$, which implies

$$
\left(\frac{2 l-5}{2 l}+o(1)\right) \sum_{j=1}^{l} \widetilde{N}_{0}\left(r, \frac{1}{f_{1}-\alpha_{j}}\right) \leq\left(\frac{5}{2 l}+o(1)\right) \sum_{j=1}^{l} \tilde{N}_{0}\left(r, \frac{1}{f_{2}-\alpha_{j}}\right)
$$

for $r \notin E$. This leads to

$$
\liminf _{r \rightarrow+\infty} \frac{\sum_{j=1}^{l} \tilde{N}_{0}\left(r, \frac{1}{f_{1}-\alpha_{j}}\right)}{\sum_{j=1}^{l} \widetilde{N}_{0}\left(r, \frac{1}{f_{2}-\alpha_{j}}\right)} \leq \frac{5}{2 l-5}
$$

which is a contradiction with the assumption of Theorem 2.2. Thus, $f_{1} \equiv f_{2}$.

Therefore, this completes the proof of Theorem 2.2.

\section{Acknowledgements}

We thank the referee(s) for reading the manuscript very carefully and making a number of valuable and kind comments which improved the presentation.

\section{Funding}

This work was supported by the National Natural Science Foundation of China (11561033, 11761035), the Natural Science Foundation of Jiangxi Province in China (20181BAB201001, 20171BAB201002, 20151BAB201008), and the Foundation of Education Department of Jiangxi (GJJ180734) of China. The third author was supported by CSC 2019.

\section{Availability of data and materials}

No data were used to support this study.

\section{Competing interests}

The authors declare that they have no competing interests.

\section{Authors' contributions}

The main idea of this paper was proposed by HYX. HYX prepared the manuscript initially and performed all the steps of the proofs in this research. All authors read and approved the final manuscript.

\section{Author details}

${ }^{1}$ School of Mathematics and Computer Science, Shangrao Normal University, Shangrao, China. ${ }^{2}$ Department of Mathematics \& Computer Science, Jiangxi Science \& Technology Normal College, Nanchang, China.

\section{Publisher's Note}

Springer Nature remains neutral with regard to jurisdictional claims in published maps and institutional affiliations.

Received: 28 February 2019 Accepted: 1 November 2019 Published online: 09 November 2019

\section{References}

1. Hayman, W.K.: Meromorphic Functions. The Clarendon Press, Oxford (1964)

2. Yang, L.: Value Distribution Theory. Springer, Berlin (1993)

3. Yi, H.Y.: Uniqueness Theory of Meromorphic Functions. Kluwer Academic, Dordrecht (2003)

4. Nevanlinna, R.: Le Théorème de Picard-Borel et la Théorie des Fonctions Méromorphes. Reprinting of the 1929 Original. Chelsea, New York (1974)

5. Chang, X.K., Liu, S.Y., Zhao, P.J., Li, X.: Convergent prediction-correction-based ADMM for multiblock separable convex programming. J. Comput. Appl. Math. 355, 270-288 (2018)

6. Chang, X.K., Liu, S.Y., Zhao, P.J., Song, D.: A generalization of linearized alternating direction method of multipliers for solving two-block separable convex programming. J. Comput. Appl. Math. 357, 251-272 (2019) 
7. Ru, M.: The recent progress in Nevanlinna theory. J. Jiangxi Norm. Univ. Nat. Sci. 42, 1-11 (2018)

8. Tu, J., Pu, S.F., Rao, D.F.: The composition of meromorphic function and entire function of finite logarithmic order. J. Jiangxi Norm. Univ. Nat. Sci. 42, 587-591 (2018)

9. $\mathrm{Xu}, \mathrm{Y}$.: Some generalizations and improvements of montel's criterion. J. Jiangxi Norm. Univ. Nat. Sci. 43, 122-127 (2019)

10. Fang, M.L.: Uniqueness of admissible meromorphic functions in the unit disc. Sci. China Ser. A 42, 367-381 (1999)

11. Zheng, J.H.: Value Distribution of Meromorphic Functions. Springer, Beijing (2010)

12. Cao, T.B., Yi, H.X., Xu, H.Y.: On the multiple values and uniqueness of meromorphic functions on annuli. Comput. Math. Appl. 58, 1457-1465 (2009)

13. Khrystiyanyn, A.Y., Kondratyuk, A.A.: On the Nevanlinna theory for meromorphic functions on annuli. i. Mat. Stud. 23 19-30 (2005)

14. Khrystiyanyn, A.Y., Kondratyuk, A.A.: On the Nevanlinna theory for meromorphic functions on annuli. ii. Mat. Stud. 24, 57-68 (2005)

15. Kondratyuk, A.A., Laine, I.: Meromorphic functions in multiply connected domains. In: Laine, I. (ed.) Fourier Series Methods in Complex Analysis. Proceedings of the Workshop, Mekrijärvi, Finland, July 24-29, 2005, pp. 9-111 (2006)

16. Korhonen, R.: Nevanlinna theory in an annulus, value distribution theory and related topics. Adv. Complex Anal. Appl. 3, 167-179 (2004)

17. Lin, W.C., Mori, S., Tohge, K.: Uniqueness theorems in an angular domain. Tohoku Math. J. 58, 509-527 (2006)

18. Liu, H.F., Mao, Z.Q.: On uniqueness of meromorphic functions sharing five small functions in some angular domains. Taiwan. J. Math. 17, 1779-1790 (2015)

19. Liu, H.F., Mao, Z.Q.: Meromorphic functions in the unit disc that share slowly growing functions in an angular domain. Comput. Math. Appl. 62, 4539-4546 (2011)

20. Zheng, J.H.: On uniqueness of meromorphic functions with shared values in some angular domains. Can. J. Math. 47, $152-160$ (2004)

21. Zheng, J.H.: On uniqueness of meromorphic functions with shared values in one angular domains. Complex Var. Elliptic Equ. 48, 777-785 (2003)

22. Xu, H.Y., Xuan, Z.X.: The uniqueness of analytic functions on annuli sharing some values. Abstr. Appl. Anal. 2012(1), 309 (2012)

23. Xu, H.Y., Cao, T.B.: Uniqueness of two analytic functions sharing four values in an angular domain. Ann. Pol. Math. 99(1), 55-65 (2010)

24. Lahiri, I.: Weighted sharing and uniqueness of meromorphic functions. Nagoya Math. J. 161, 193-206 (2001)

25. Lahiri, l.: Weighted value sharing and uniqueness of meromorphic functions. Complex Var. Theory Appl. 46, 241-253 (2001)

26. Xu, H.Y., Wang, H.: Results on meromorphic functions partially sharing some values in an angular domain. Mathematics 6, 297 (2018)

27. Ishizaki, K.: Meromorphic functions sharing small functions. Arch. Math. 77, 273-277 (2001)

28. Li, B.Q.: Uniqueness of entire functions sharing four small functions. Am. J. Math. 119, 841-858 (1997)

29. Li, X.M., Yi, H.X.: Uniqueness of meromorphic functions whose nonlinear differential polynomials share one value or have the same fixed points in an angular domain. Acta Math. Sci. 34, 593-609 (2004)

30. Li, Y.H., Qiao, J.Y.: The uniqueness of meromorphic functions concerning small functions. Sci. China Ser. A 43, 581-590 (2000)

31. Yi, H.X.: On one problem of uniqueness of meromorphic functions concerning small functions. Proc. Am. Math. Soc 130, 1689-1697 (2002)

32. Yi, H.X., Li, Y.H.: Meromorphic functions that share four small functions. Chin. Ann. Math., Ser. A 22, 271-278 (2001)

33. $\mathrm{Xu}$, H.Y., Liu, S.Y.: The uniqueness of meromorphic functions in k-punctured complex plane. Open Math. 15, 724-733 (2017)

34. Xu, H.Y., Liu, S.Y., Wang, H.: On meromorphic functions for sharing two sets and three sets in $m$-punctured complex plane. Open Math. 14, 913-924 (2016)

35. Hanyak, M.O., Kondratyuk, A.A.: Meromorphic functions in m-punctured complex planes. Mat. Stud. 27, 53-69 (2007)

\section{Submit your manuscript to a SpringerOpen ${ }^{\circ}$ journal and benefit from:}

- Convenient online submission

- Rigorous peer review

- Open access: articles freely available online

- High visibility within the field

- Retaining the copyright to your article

Submit your next manuscript at springeropen.com 